\title{
L'ordre d'opération dans l'Armée de Terre
}

Étude d'un genre normé

\section{Jean-Louis Trouillon}

\section{OpenEdition}

\section{Journals}

Édition électronique

URL : https://journals.openedition.org/asp/1288

DOI : $10.4000 /$ asp. 1288

ISBN : 978-2-8218-0392-3

ISSN : 2108-6354

\section{Éditeur}

Groupe d'étude et de recherche en anglais de spécialité

\section{Édition imprimée}

Date de publication : 1 mai 2003

Pagination : 47-57

ISSN : 1246-8185

\section{Référence électronique}

Jean-Louis Trouillon, «L'ordre d'opération dans l'Armée de Terre », ASp [En ligne], 39-40 | 2003, mis en ligne le 01 mai 2003, consulté le 21 septembre 2021. URL : http://journals.openedition.org/asp/1288 ; DOI : https://doi.org/10.4000/asp.1288

Ce document a été généré automatiquement le 21 septembre 2021.

Tous droits réservés 


\title{
L'ordre d'opération dans l'Armée de Terre
}

\author{
Étude d'un genre normé
}

Jean-Louis Trouillon

\section{Introduction}

1 Le concept même d'ordre et en particulier d'ordre verbal, du type Attention! / Forward, march! / Left, right, left right! ou encore Stand at ease!, mais ce ne sont là que quelques exemples, apparaît souvent aux yeux du public non spécialiste comme une des caractéristiques principales du discours militaire.

2 Les ordres dans le milieu militaire appartiennent à deux types majeurs : d'une part les ordres figés, codifiés, semblables à ceux énoncés supra, et que l'on pourrait qualifier de routiniers, et d'autre part les ordres originaux conçus en préparation ou en réponse à une situation particulière.

3 Cette étude portera sur le représentant majeur de cette deuxième catégorie, l'ordre d'opération dans l'Armée de Terre ${ }^{1}$. La première catégorie ne relève en effet que du rituel, il s'agit d'énoncés presque uniquement oraux, de stimuli entraînant systématiquement pour chacun d'entre eux un seul et unique type de réponse physique, un mouvement particulier accompli par le(s) destinataire(s) de l'ordre en question. Leur étude est donc relativement simple même si l'on peut procéder à leur sujet à des observations intéressantes, en particulier quant à la segmentation en syllabes ou aux déplacements d'accent tonique par rapport aux prononciations reconnues dans le milieu civil2.

4 La deuxième catégorie, bien qu'assez fortement codifiée ainsi qu'il sera montré au cours de cette étude, est plus ouverte : tout en respectant la mission impartie l'auteur dispose d'une certaine marge de rédaction car il lui faut rédiger l'ordre en fonction de nombreux paramètres, aucune situation n'étant jamais identique à une autre. Le milieu physique dans lequel se déroulera l'action, la chronologie impartie, les conditions météorologiques, 
l'état des matériels, la disponibilité des appuis, la force et l'attitude de l'ennemi, sont des variables que l'on retrouvera mentionnées sous une forme ou une autre dans les ordres.

\section{Qu'est-ce qu'un ordre d'opération?}

De nombreux films de guerre ont popularisé l'image d'officiers penchés sur des maquettes recouvertes de pictogrammes et de modèles réduits divers : ces objets sont les représentations à l'échelle d'une part des données physiques du terrain et d'autre part des situations tactiques et des décisions que ces situations ont entraînées. Même si de tels modèles physiques existent réellement (connus sous le nom de caisse à sable ou sandbox) ils sont surtout utilisés de nos jours lors de l'instruction en temps de paix et sont d'ailleurs de plus en plus souvent remplacés par des images virtuelles sur écrans d'ordinateurs. Le choix assez systématique de ces maquettes par le cinéma est certainement dû à leur force de représentation visuelle plus évocatrice qu'un document écrit ou qu'une simple carte.

6 La réalité opérationnelle est quelque peu différente : si les situations tactiques et les décisions prises sont effectivement souvent représentées visuellement par des ordres graphiques (Overlay Orders, Overlay Os ou OVOs), calques superposés aux cartes dites d'ÉtatMajor, ces ordres sont de simples schémas renseignés (Cf. Annexe 1) accompagnant en fait des instructions plus ou moins développées selon le moment de la manoeuvre et l'unité destinataire.

\subsection{Les différentes formes d'ordre}

7 Ces instructions portent des noms différents, c'est ainsi que l'on peut distinguer les catégories suivantes :

- les Oral Orders (0Os), qui sont les seuls à être intégralement oraux;

- les Operation Orders (OPOs chez les Britanniques et OPORDs chez les Américains ou pour l'OTAN), généralement uniquement écrits mais qui sont souvent accompagnés de graphiques et peuvent être partiellement oralisés ;

- les Warning Orders (WNGOs), «ordres préparatoires ${ }^{3}$, qui précèdent généralement les ordres d'opération;

- les Movement Orders (MOVOs), «ordres de marche» ou «de mouvement», annexes aux ordres d'opération ou logistiques;

- les Fragmentary Orders (FRAGOs), «ordres de conduite » ${ }^{4}$, qui sont des ordres d'opération simplifiés s'adressant soit à l'ensemble des unités avant que l'ordre d'opération ne soit entièrement rédigé, soit à des unités qui n'ont pas à connaître la totalité des ordres, soit encore, et c'est le cas le plus fréquent, pour modifier un ordre d'opération en cours d'exécution pour tenir compte d'un changement de situation par rapport aux prévisions initiales. Lors de la récente guerre d'Irak, le Général Tommy Franks a en effet rappelé the old military adage selon lequel war plans never survive the first battle.

Il existe également des ordres particuliers pour certaines armes ou services, par exemple pour la logistique: Combat Service Support Order chez les Britanniques (CSSO), Administrative Order (ADMINO) aux États-Unis, Logistic Order (LOGORD) dans le cadre de l'OTAN ${ }^{5}$. 
9 L'ordre d'opération est le plus complet de tous. Les autres n'en sont, à des degrés divers, que des formes simplifiées, qui en respectent la structure. L'ordre d'opération peut être accompagné d'un ordre graphique (cf. supra), qui illustre particulièrement l'un des paragraphes de l'ordre d'opération, à savoir le mode d'action retenu par le commandement.

\subsection{La structure des ordres}

Quelle que soit leur origine nationale, au sein de l'ensemble des forces de l'OTAN les ordres obéissent tous en effet à un certain nombre de normes quant à leur structure interne et leur présentation. On trouve, toujours selon le même agencement (cf. Annexe 2),

- d'abord les renseignements purement administratifs (avec en tête le degré de protection suivi du nombre d'exemplaires, du nombre total de pages et des références de l'autorité d'émission), les références horaires du moment d'émission, puis les références cartographiques et horaires correspondant à la situation donnée,

- le premier paragraphe, qui présente la situation tactique elle-même, avec au moins deux sous-paragraphes pour la situation ennemie d'abord puis la situation amie, ainsi qu'il est précisé dans le manuel américain d'État-Major :

Give briefly the general picture, so that subordinate commanders will understand the current situation, under the following headings.

a. Enemy Forces. Give composition, disposition, location, movements, estimated strengths, identification, and capabilities. Summarize the enemy situation in the intended area of operations. References may be made to other record documents that amplify information included here.

b. Friendly Forces. Give information on friendly forces that may directly affect the action of subordinate commanders. These forces include those not attached or organic to the command for the contemplated operation, but whose presence on a flank or other adjacent area is of interest. Include information on such forces that subordinate commanders need to know to accomplish their tasks ${ }^{6}$.

- le deuxième paragraphe, qui donne la mission de l'unité recevant l'ordre,

- le troisième paragraphe, qui détaille le mode d'action décidé par l'autorité pour l'unité recevant l'ordre,

- les quatrième et cinquième paragraphes, qui décrivent les moyens spécifiques mis en œuvre en particulier au niveau de la logistique et des transmissions,

- la demande d'accusé de réception,

- le nom et le grade du rédacteur de l'ordre,

- les annexes,

- la liste des destinataires de l'ordre.

11 Enfin, comme pour tout autre document militaire, on trouve un rappel en bas de page du degré de protection.

Des documents préformatés existent bien entendu afin d'accélérer la rédaction et d'éviter la répétitivité de tâches routinières. L'Armée de Terre des États-Unis a développé l' Electronic Operation Order (OPORD) : «The Electronic OPORD is a rapid staff planning tool that provides Microsoft Word (MS Word) templates for all parts of a notional OPORD $»^{7}$. 


\subsection{Les normes de rédaction des ordres}

13 Pour ce qui est de la rédaction elle-même, des normes existent également, dont on trouvera un exemple britannique en Annexe 3. Les indications sont très précises et touchent aussi bien à la structure d'ensemble («It may be necessary to start this paragraph with a brief introduction ») qu'à la graphie (" paragraph headings [...] are not abbreviated, are written in capital letters and not followed by a full stop »)ou à la syntaxe ("Verbs in 'Friendly Forces' are to be in present tense »). Elles font l'objet de nombreux cours d'application en école d'État-Major (Staff College). Chez les Britanniques, par exemple, il est rappelé dans l'aide-mémoire de l'officier d'État-Major, que "The main rule is that in operational writing the maximum use is to be made of abbreviations and the text is to be written in note form to save time and space, remembering always that the meaning remains clear $»^{8}$.

L'extrême précision contraignante des instructions, qui rappelle bien souvent les conseils du MLA Handbook for Writers of Research Papers quant au choix de tel ou tel signe de ponctuation, ou encore pour ce qui relève du rôle joué par les majuscules, aboutit de fait à une codification : elle est destinée à éviter toute ambiguïté, elle permet un gain de temps lors de la rédaction ${ }^{9}$ puisqu'il n'y a, en fonction d'une manœuvre donnée, qu'un nombre prédéfini de termes possibles même si l'on trouve parfois de légères variantes ou des intrusions surprenantes ${ }^{10}$.

\subsection{Le processus d'élaboration des ordres}

L'ordre d'opération est l'aboutissement d'un long processus codifié d'observations, de recherches, d'interprétation, d'analyse, de synthèse et d'hypothèses. C'est la partie Estimate du Decision making process de l'OTAN ${ }^{11}$.

Ces différentes démarches représentent des phases soit consécutives soit simultanées accomplies par les membres de l'État-Major (Staff). Ces membres sont en nombre plus ou moins important selon le niveau de l'unité émettrice ${ }^{12}$, mais la rédaction finale des décisions et des choix opérés in fine par le Chef d'État-Major est, dans tous les cas, considérée comme l'œuvre d'une seule personne dont on trouve le nom à la fin de l'ordre.

Les traces de ces différentes phases se retrouvent de façon plus ou moins nette dans la rédaction de l'ordre qui représente la synthèse de l'ensemble du processus.

La phase préliminaire à toute décision est l'étude du terrain sur lequel va se dérouler l'opération. L'examen de cette zone va aboutir à la réalisation d'un "chevelu », carte physique montrant le modelé principal du terrain, accompagné d'une fiche synthétique donnant les caractéristiques physiques et humaines de la zone retenue pour l'action considérée : habitat et infrastructure surtout, en insistant sur les voies de communication et les obstacles éventuels au déplacement.

De cet ensemble, les concepteurs de la manœuvre déduiront d'abord les grands axes de cette dernière, mais le rédacteur de l'ordre ne retiendra en fin de compte que des ordres pour action du genre secure hill 103 ou screen enemy advance between river $X$ and crossroads $Y$ sans préciser que la côte 103 est un point d'observation essentiel pour une autre unité ou encore que le relief de la zone comprise entre la rivière $\mathrm{X}$ et le croisement $\mathrm{Y}$ se prête tout particulièrement au jalonnement (screening): chaque subordonné recevant l'ordre possède en effet la carte de la zone et toute explication serait redondance d'information; d'autre part, une telle explicitation relèverait d'une justification des choix, ce qui n'est 
pas prévu dans ce cadre : le subordonné recevant l'ordre n'a pas à discuter la mission qui lui est confiée par l'autorité supérieure, et ce de par la nature même de l'institution ${ }^{13}$.

\section{L'ordre d'opération et le chercheur en langue de spécialité}

\subsection{Le non-dit} unités ennemies : régiments de chars ( $T$ k regts) et bataillons autonomes de chars (separate tk bns) des $80^{\mathrm{e}}$ et $94^{\mathrm{e}}$ Divisions de Fusilliers Motorisés (80 and 94 MRDs), la connaissance des structures de l'armée ennemie a permis de poser leur présence comme certaine, et la connaissance de la doctrine ennemie et de l'ordre de bataille adverse a permis de poser l'hypothèse de leur présence en un certain lieu. Comme dans l'exemple du paragraphe précédent, l'ensemble du processus est absent de l'ordre, le destinataire connaissant lui aussi les structures des forces ennemies, il comprend le raisonnement sans qu'il soit nécessaire de le lui rappeler.

\subsection{Des variations lexicales selon l'origine}

L'on a pu remarquer dans le paragraphe 1.1 la présence d'appellations parfois différentes selon qu'il s'agissait de documents américains, britanniques ou OTAN. L'équivalent français tient parfois lui-même compte de ces différences, le français de France n'est pas toujours le même que le français de l'OTAN qui doit tenir compte des influences belges! C'est là un point qu'il est bon de garder en mémoire: le secteur LANSAD a en effet tendance à parler $\mathbf{d u}$ discours des mathématiques, $\mathbf{d u}$ discours de l'économie, du discours de la géologie, etc., et cela est justifié par la recherche car, pour un domaine donné, le substrat du discours est identique. Toutefois, une des caractéristiques fondamentales du secteur LANSAD étant la transmission de la langue spécialisée, il est bon de s'attacher aussi aux réalisations de surface qui peuvent évoluer dans le temps comme dans l'espace : ces formes apparentes sont celles que donnent le lexique et la syntaxe, elles intéressent 
donc au premier chef les apprenants. Si le discours militaire anglophone semble bien, de par son caractère injonctif, mais parfois aussi rituel ou didactique, présenter une forte unité, les apparences qu'il revêt reflètent aussi son origine nationale (ou supranationale lorsqu'il s'agit de documents OTAN ou ONU).

\subsection{Des variations dans la rédaction selon l'origine}

On constate chez les Britanniques, cela a déjà été mentionné, une très haute fréquence d'abréviations de diverses catégories lexicales ainsi que de tournures elliptiques, en particulier de formes passives. On peut à cet effet comparer un sous-paragraphe sur l'ennemi :

1 (UK) Armd Div probably faced by elms 80 MRD and 94 MRD. Poss inter div bdry on 71 Easting. Up to two MRR, both at approx $60 \%$ str, are not yet fully prep in posns along line BORDON (SU8035)-MEDSTEAD (SU6536). Tk regts and separate tk bns of 80 and 94 MRDs not yet loc, but assumed to be south of BASINGSTOKE ${ }^{15}$.

à un sous-paragraphe équivalent américain (la situation tactique est bien entendu différente mais ce n'est pas là ce qui explique les différences de rédaction). Ce dernier est un texte intégralement rédigé, qui fait certes appel à quelques sigles ou abréviations mais uniquement dans le cas d'unités (division, brigade, bataillon) ou de matériels (canons ZU-23-2 et systèmes de missiles SA-7 GRAIL) :

The 1st Inf Div (ATL) is currently operating at an estimated strength of 50\% in both personnel and equipment. The 6th Inf Bde (ATL) is assessed to be at $60 \%$ strength in all areas. The 6th Inf Bde (ATL) does have two light Inf Bns and one Bn of Mechanized (BMP) Inf. Indirect fire assets consist of ZU-23-2 and SA-7 GRAIL systems.

Les ordres OTAN ou ONU sont également rédigés selon les formes américaines reflétant ainsi la place prépondérante des États-Unis au sein de ces deux organisations. Compte tenu de la diversité des nationalités et donc des langues parlées par les différents membres de ces organisations, on peut également poser l'hypothèse d'une plus grande lisibilité d'un ordre intégralement rédigé.

\subsection{Des variations dues à la technique de transmission} face ou par radio, le texte écrit, éventuellement transmis par fax mais la tendance actuelle est à la numérisation du champ de bataille.

La transmission par message radio n'entraîne qu'une modification mineure : l'ensemble du texte écrit est saisi en majuscules sur les formulaires appropriés, comme pour tout autre message radio, et ce afin d'améliorer la lisibilité du texte lors de la lecture orale par le transmetteur.

Le fax, puis l'utilisation de l'informatique, ont en revanche entraîné une profonde modification dans l'utilisation des symboles utilisés dans les ordres graphiques afin d'éviter toute erreur d'interprétation. 
31 Les symboles désignant les unités ennemies étaient traditionnellement dessinés en rouge ou, lorsque les couleurs n'étaient pas disponibles (cas de photocopies), comportaient un double trait ( $C f$. la partie droite de l'Annexe 1 pour une illustration de ce double trait). Les couleurs ne pouvaient être transmises par fax et ce média brouillait aussi parfois le double trait.

32 L'utilisation d'écrans informatiques monochromes présentant le même inconvénient il a été décidé de modifier la forme même des symboles qui ne sont plus des rectangles (forme conservée pour toutes les unités amies) mais des hexagones.

\subsection{Des variations dans le temps}

33 Si l'on se place sous l'angle de l'analyse du discours il est possible de distinguer deux types majeurs d'ordres d'opérations: d'une part tous ceux qui relèvent de situations militaires traditionnelles (défense, attaque, couverture, reconnaissance, etc.), et d'autre part tous ceux qui, depuis la dernière décennie du XXème siècle surtout, correspondent aux nouveaux objectifs assignés aux forces armées.

Les forces armées occidentales sont en effet de plus en plus souvent chargées d'opérations de maintien, d'imposition ou de restauration de la paix. Le Timor oriental ou l'ex-Yougoslavie en sont des exemples récents.

35 Cette définition de nouveaux objectifs, qui implique une nouvelle vision de la fonction militaire, entraîne aussi des modifications de la présentation du contenu des ordres d'opération. Dans le premier cas, ainsi que le montre le document d'exercice britannique suivant qui détaille les forces ennemies (En forces), le sous-paragraphe sur l'ennemi présente la composition, la force, l'identification, la position, les capacités, les intentions supposées de cet ennemi :

\section{Situation}

a. En forces. Addl to 1 (UK) Armd Div PICINTSUM 3/95:

1) FLET no further south than 30 Northing.

2) En MDA estb along line GUILDFORD (SU9950) to ANDOVER (SU3745) with three MRDs fwd: 79 MRD west, 80 MRD centre, 94 MRD east.

3) 1 (UK) Armd Div probably faced by elms 80 MRD and 94 MRD. Poss inter div bdry on 71 Easting. Up to two MRR, both at approx $60 \%$ str, are not yet fully prep in posns along line BORDON (SU8035)-MEDSTEAD (SU6536). Tk regts and separate tk bns of 80 and 94 MRDs not yet loc, but assumed to be south of BASINGSTOKE.

4) $37 \mathrm{TD}$ is still loc north of River Thames as a result of cont success of Allied air interdiction.

Lors d'une mission de paix ce paragraphe disparaît sous cette forme au profit d'un exposé de la situation politique locale ainsi que le montre un document OTAN de la SFOR, force de stabilisation en Bosnie :

1.Situation

A. Two OSCE Article II inspections will take place on 9 June 97. The first inspection will be a team from the Republic of SPRSKA conducting an inspection of two federation declared sites. The second inspection will be a team from the Federation of BIH inspecting two sites in the Republic of SPRSKA.

B. As of this time neither team has declared which sites will be inspected. As 
soon as the information is made available it will be disseminated to the MNDs. be...ing) pour les certitudes avérées mais on peut découvrir d'autres formes : Cuban MIG 21s have been sighted in $\mathrm{XXXX} .{ }^{17}$ Il fait appel à divers procédés pour l'expression des hypothèses selon le degré de certitude de ces dernières : probably, possible, assessed to be, assumed to be, should, at approximately, et ce quelle que soit l'origine nationale ou supranationale de l'ordre.

41 Le sous-paragraphe friendly forces est également descriptif au temps présent pour tout ce qui relève de la description de la situation.

42 Les prescriptions peuvent revêtir plusieurs formes que l'on retrouvera également dans le paragraphe Task organisation and mission:

- infinitif avec to : to provide a casualty evacuation helicopter if and when required ${ }^{18}$;

- WILL : SFOR will employ MND(N) to coordinate and conduct assessments ${ }^{19}$; WILL se retrouvera fréquemment en conjonction avec des passifs : each supply truck will be directed to the support area as soon as empty ${ }^{20}$;

- des impératifs sont également présents, en particulier avec la forme figée be prepared to ${ }^{21}$ : be prepared to deal with bypassed enemy positions ${ }^{22}$;

- BE TO : the ARRC is to attack north into Obj NEPTUNE ${ }^{23}$; il est à noter qu'une simple lecture d'ordres d'opération permet de repérer une fréquence d'emploi élevée de BE TO ;

- de manière plus surprenante, il sera fréquemment fait appel au temps présent pour anticiper d'une action future ainsi qu'en témoigne cet extrait s'adressant au $66^{\mathrm{e}}$ bataillon du $2^{\mathrm{e}}$ Régiment d'Infanterie : on order 2-66 Inf conducts supporting attack in zone to clear zone of ...24

Le sous-paragraphe Concept of Operation du paragraphe 3 (Execution) est le seul qui donne directement la parole au Chef d'État-Major responsable de l'ordre en dernier ressort : My intent is to quickly destroy ... ${ }^{25}$ 


\section{Conclusion}

\section{BIBLIOGRAPHIE}

Electronic Operation Order <https://www.doctrine.quantico.usmc.mil/ opordweb/opord.pdf>.

Joint Staff Officers Guide AFSC Pub 1-1997. Norfolk, Va : AFSC.

Gibaldi, Joseph. 1995. MLA Handbook for Writers of Research Papers, 4e éd. New York : The Modern Language Association of America.

Staff Officers' Handbook, Army Code Number 71038, Issue 1.1: July 2000, p.5-1-1.

Méthode d'élaboration d'une décision opérationnelle, 20 juillet 2001. n953/CDES/CREDAT B.1.

NATO Guidelines for Operation Planning. January 2001.

NATO Military Symbols for Land Based Systems. 2000. STANAG 2019, APP6A.

Formats for Orders and Designation for Timings Location of Boundaries. October 2000. STANAG 2014 Edition 9.

NATO Glossary of Terms and Definitions. December 2001. STANAG 3680, AAP6.

ANNEXES

\section{Annexe 1}




\section{Exemple d'ordre graphique américain de niveau Corps d'armée}

Figure 3-1. Corps concept of operation: the deliberate attack (source FM71-100-2 Infantry Division operations 1993 <http://www.scribd.com/doc/12885610/Operations-and-Tatics>.

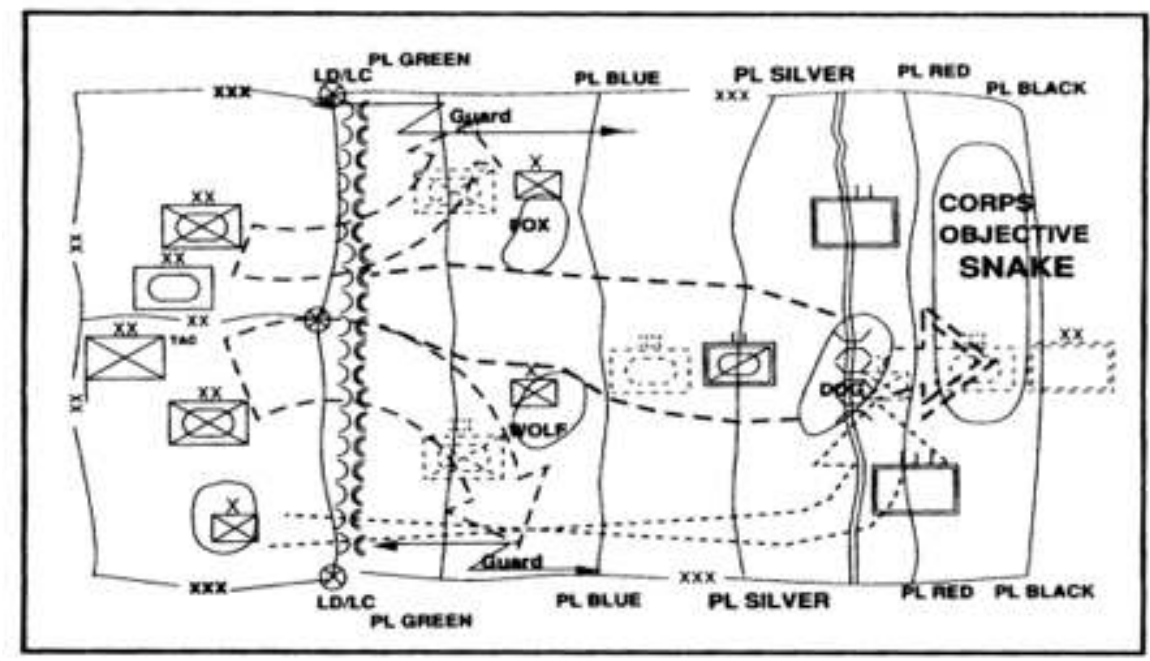

Annexe 2

\section{Structure d'un ordre d'opération}

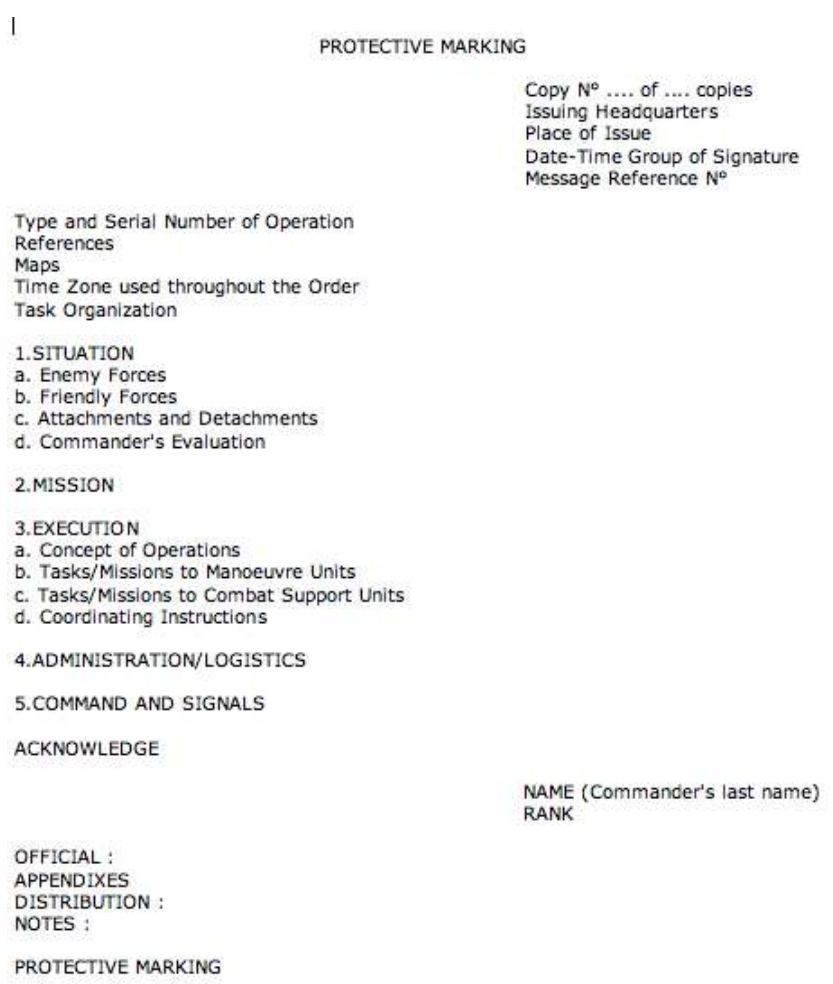

\section{Annexe 3}

Normes britanniques de rédaction pour le paragraphe Enemy Forces

Afin d'habituer les stagiaires d'État-Major aux abréviations le manuel d'instruction luimême fait largement appel à ces dernières :

En Forces. Incl such info as composn, disposn, locs, est str, ident and capabilities. En air 
activity and nuc capability should be incl if recipients need to know. It may be sufficient to ref to a separate INTSUM already issued or to fol: alt the info may be incl in an annex.

Le paragraphe développé doit être compris ainsi :

Enemy Forces. Include such information as composition, disposition, locations, estimated strength, identification and capabilities. Enemy air activity and nuclear capability should be included if recipients need to know. It may be sufficient to refer to a separate INTSUM (Intelligence Summary) already issued or to follow: alternatively the information may be included in an annex.

\section{NOTES}

1. Une précision d'ordre sémantique à propos de la polysémie du mot : si «ordre » sous-entend parfois l'idée d'organisation, et l'on en trouve aussi des exemples dans le discours militaire, comme par exemple « ordre de bataille ", order of battle, ou encore l'idée d'harmonie ou de règle comme dans "ordre public », et bien d'autres sens encore, il ne sera ici question que de l'ordre de type prescriptif.

2. Attention! par exemple se prononce généralement en deux syllabes, soit ten'shun soit ten'hut. L'accent principal a tendance à disparaître de stand dans Stand at ease!; at reste réduit et on ne trouve qu'un seul accent portant sur ease.

3. Parfois « ordres d'avertissement » lorsqu'il s'agit d'un document OTAN.

4. Parfois « ordres simplifiés » lorsqu'il s'agit d'un document OTAN.

5. On parle en français $d^{\prime}$ " ordre administratif et logistique » ou « OAL ».

6. <http://www.fas.org/man/dod-101/dod/docspub1_97/APPENH.html>

7. Electronic Operation Order <https://www.doctrine.quantico.usmc.mil/ opordweb/opord.pdf>

8. Pour un exemple d'abréviations, on se reportera infra au paragraphe 2.3.

9. Outre le gain de temps à la rédaction, les abréviations permettent une structure plus dense et donc une longueur de document réduite ce qui entraîne une réduction du nombre de photocopies, mais aussi un temps de transmission inférieur lorsque la transmission a lieu par fax ou par radio. A fortiori en graphie morse, même si ce moyen n'est plus guère utilisé.

10. Dans un ordre d'opération, français il est vrai, au lieu du « défendre la ville $X$ » qui aurait été la norme, le rédacteur de l'ordre avait écrit « défendre avec pugnacité la ville X ». Il serait sans doute intéressant, après avoir établi un corpus, de rechercher ce type de variation, et, partant de là, de se poser la question éventuelle du hedging.

11. Ce processus porte en français le nom de «MEDO, Méthode d'élaboration d'une décision opérationnelle ", méthode enseignée en École d'État-Major.

12. Les niveaux principaux sont, par ordre décroissant de taille : Armée, Corps d'Armée, Division, Brigade, Régiment.

13. Dans les manuels d'État-Major français on trouve la phrase très explicite : «La mission est sacrée ».

14. Le bien connu Deuxième Bureau.

15. Ce paragraphe est à lire : "The 1st UK Armoured Division is probably faced by elements of the 80th Motorized Rifle Division and the 94th Motorized Rifle Division. The inter division boundary may be on 71 Easting. Up to two Motorized Rifle Regiments, both at approximately $60 \%$ strength, are not yet fully prepared in positions along the line BORDON (SU8035)-MEDSTEAD (SU6536). The tank regiments and separate tank battalions of the 80th and 94th Motorized Rifle Divisions have not yet been located, but are assumed to be south of BASINGSTOKE. »

16. Il y a quelques années, dans l'armée française on utilisait parfois l'expression «le primo alpha » pour désigner l'ennemi puisque l'ennemi était toujours décrit dans le paragraphe 1 , lu 
primo, et dans le sous-paragraphe a, lu alpha dans l'alphabet militaire. La métaphore, si elle existait encore, n'aurait plus nécessairement de nos jours le même référent.

17. Ordre américain BUFORD, non daté.

18. Ordre OTAN du 10 décembre 1998.

19. Ordre OTAN du 5 mars 1999.

20. Ordre d'exercice américain, non daté.

21. Correspond au français «en mesure de» que l’on trouve sous la forme écrite abrégée « emd ».

22. Ordre d'exercice américain, non daté.

23. Ordre d'exercice britannique 1/95 de 1(UK) Armd Div, $1^{\mathrm{e}}$ Division Blindée, du 18 avril 1995.

24. Ordre d'exercice américain BUFORD, non daté.

25. Ordre d'exercice américain BUFORD, non daté. L'équivalent français est également personnel : « Je veux ... ».

26. Plusieurs de ces leçons sont consultables sur l'Internet. Le Center for Army Lessons Learned de l'Armée de Terre des États-Unis se trouve à l'adresse <http://call.army.mil/>.

\section{RÉSUMÉS}

L'article étudie les caractéristiques majeures de l'ordre d'opération qui permettent de le définir en tant que genre: structure figée, normes de rédaction strictes, relations de l'auteur au destinataire.

The paper studies the major characteristics of the operation order to be defined as a genre with its fixed structure, strict writing norms, relations between author and addressee.

\section{INDEX}

Mots-clés : anglais militaire, genre, norme, ordre d'opération

Keywords : military English, norm, operation order

\section{AUTEUR}

\section{JEAN-LOUIS TROUILLON}

Jean-Louis Trouillon est maître de Conférences HDR à l'Université de Perpignan. Il est également instructeur en anglais militaire à l'École d'Application de l'Infanterie à Montpellier. Ses recherches portent principalement sur le lexique spécialisé et la didactique de l'anglais de spécialité. Il est l'auteur de plusieurs didacticiels en anglais scientifique. jeanlouis.trouillon@wanadoo.fr 